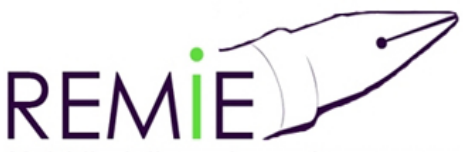

Multidisciplinary Journal of Educational Research

\section{Hipatia Press}

www.hipatiapress.com

Instructions for authors, subscriptions and further details:

http://remie.hipatiapress.com

\title{
Parentalidad Positiva y Formación Experiencial: Análisis de los Procesos de Cambio Familiar
}

Susana Torío López, José Vicente Peña Calvo \& Omar GarcíaPérez ${ }^{1}$

1) Universidad de Oviedo, España.

Date of publication: October $15^{\text {th }}, 2015$

Edition period: October 2015 - February 2016

To cite this article: Torío López, S., Peña Calvo, J.V., \& García-Pérez, O. (2015). Parentalidad Positiva y Formación Experiencial: Análisis de los Procesos de Cambio Familiar. Multidisciplinary Journal of Educational Research, 5 (3), 296-315. doi:10.17583/remie.2015.1533

To link this article: http://dx.doi.org/10.17583/remie.2015.1533

PLEASE SCROLL DOWN FOR ARTICLE

The terms and conditions of use are related to the Open Journal System and to Creative Commons Attribution License (CC-BY). 


\section{Positive Parenting and Experiential Training: Analysis of Processes of Family Change}

Susana Torío López

University of Oviedo

Omar García-Pérez

University of Oviedo

\author{
José Vicente Peña Calvo \\ University of Oviedo
}

\section{Abstract}

Several changes are affecting families and usually cause their vulnerability as they feel unable to cope with everyday situations with accurate answers. Exchanging views and sharing experiences with other parents may help them to succeed in these new situations. "Building the everyday" is a parental education program, which focuses on the promotion of positive parenting. The program tries to improve educational outcomes through better sharing of family responsibilities and with a supportive and strong sense of community. 48 fathers and mothers whose children were in Primary Education in 6 schools of the Principality of Asturias have participated in the study (22 couples and 4 monoparental families). The aim of the present study is to show the results of the final evaluation of the program and to assess the effects of experiential programs in the processes of family change. The results show a great interest in participants in the program and its structure; they also show changes in parents' attitudes, preferences and beliefs about gender roles; the program improves the division of domestic work and other areas such as communication, the establishment of rules and conflict resolution. In short, these processes of change, offer families learning opportunities which may enable the reconstruction / optimization of their parenthood.

Keywords: positive parenting, experiential training, corresponsibility, evaluation, intervention with families 

No. 3 October 2015 pp. 296-315

\section{Parentalidad Positiva y Formación Experiencial: Análisis de los Procesos de Cambio Familiar}

Susana Torío López

Universidad de Oviedo

Omar García-Pérez

Universidad de Oviedo
José Vicente Peña Calvo
Universidad de Oviedo

\section{Resumen}

Los cambios que están afectando al núcleo familiar provocan la indefensión de las familias ante las posibles maneras de dar respuesta a las situaciones cotidianas, siendo necesario compartir experiencias con otros padres y madres. "Construir lo cotidiano" es un programa de educación parental dirigido a favorecer la parentalidad positiva y mejorar los resultados educativos a través de un mejor reparto de las cargas familiares con un sentido solidario y fuertemente comunitario. En el estudio han participado 48 personas (22 parejas nucleares; 4 familias monoparentales) de 6 centros educativos de Educación Infantil y Educación Primaria del Principado de Asturias. El objetivo del trabajo es mostrar resultados de la evaluación final del programa y comprobar los efectos de la formación experiencial en los procesos de cambio en la dinámica familiar. Los resultados muestran un gran interés de las familias en el planteamiento y estructura del programa, así como cambios a corto plazo en actitudes, preferencias y creencias que poseen sobre los roles sexuales, el reparto del trabajo doméstico, la comunicación, el establecimiento de normas y la resolución de conflictos. En definitiva, estos procesos de cambio, ofrecen oportunidades de aprendizaje que terminan posibilitando la reconstrucción/optimización del ejercicio de la parentalidad.

Palabras clave: parentalidad positiva, formación experiencial, corresponsabilidad, evaluación, intervención familiar 


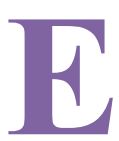

1 apoyo a la parentalidad es una tarea que ha sido destacada por el Consejo de Europa, a través de la Recomendación -REC (2006)19-, piedra angular del trabajo en el campo de la relación padres-hijos y se ha convertido en la referencia de prácticamente todas las políticas de parentalidad promovidas en Europa. La Recomendación se propone conseguir que los Estados miembros sean conscientes de la necesidad de proporcionar a los padres los mecanismos de apoyo suficientes para cumplir sus responsabilidades en la crianza y educación de sus hijos e hijas. Concretamente, entre los componentes fundamentales de las políticas y medidas propuestas (Consejo de Europa, 2006) se señala la creación de las condiciones necesarias para lograr una mejor conciliación de la vida familiar y laboral.

En este sentido, es necesario proporcionar a los padres una serie de servicios y programas centrados en el contenido de las tareas y funciones parentales (Center for Parenting and Research, 2006; Consejo de Europa, 2006; González Sánchez, 2013; Moreno, 2010; Vargas- Rubilar \& AránFilippetti, 2014) destacando, entre otros, los programas educativos y de ayuda a padres (durante el embarazo o en distintas etapas del desarrollo del niño; programas para apoyar la educación de los hijos e hijas; prevenir el abandono escolar y promover la cooperación entre padres y centros escolares, entre otros). De igual modo, en el informe "Parenting in contemporany Europe: a possitive approach" (Consejo de Europa, 2007) se señala la posibilidad que tienen los padres para aprender hablando sobre sus experiencias con otros padres, amigos y familiares. Se recomienda recurrir a servicios profesionales que de forma directa (educación para padres) e indirecta (terapia de pareja) les apoyen en su papel parental.

Entendemos por parentalidad positiva (Loizaga, 2011) el buen trato recibido por diferentes personas basado en su capacidad para querer, acoger, cuidar, calmar; en síntesis, para proteger y producir buenos tratos favoreciendo el desarrollo cerebral, cognitivo, emocional e interpersonal.

Los principios de parentalidad positiva destinados a las familias recogidos en los trabajos empíricos y programas desarrollados en el Centro de Parentalidad y Apoyo a la Familia de la Universidad de Queensland (Loizaga, 2011; March \& Orte, 2014; Sanders, 2008; Sanders, MarkieDadds \& Turner, 2003) son los siguientes: 
1. Garantizar un ambiente seguro y de interés en el que los niños puedan explorar, experimentar y desarrollar sus habilidades.

2. Crear un ambiente de aprendizaje positivo estando disponibles cuando los niños necesitan ayuda, cuidado y atención.

3. Utilizar una disciplina asertiva siendo conscientes y actuando con rapidez cuando el niño se comporta de forma inadecuada.

4. Tener expectativas realistas en relación con los hijos y uno mismo como padre o madre.

5. Cuidarse uno mismo como padre o madre satisfaciendo las necesidades personales.

En el momento actual, los cambios que están afectando al núcleo familiar (ritmos de vida, exigencias laborales de los adultos, tipología plural de familias, actitudes educativas no siempre correctas,...) provocan la indefensión de las familias ante las posibles maneras de dar respuesta a las situaciones cotidianas. El aprendizaje del rol educativo de padre y madre, aprendido por imitación de los mayores como modelo, no parece ser suficiente en nuestros días, aun cuando durante siglos, ha sido una de las fuentes más importantes de información como ayuda para la crianza y educación de nuestros menores. De igual modo, las orientaciones individuales por parte de diferentes profesionales que intervienen (pediatría, profesorado,...) son importantes, pero hay muchas situaciones en las que es necesario el intercambio de pareceres, de compartir experiencias y vivencias con otros padres y madres (buscar otras opciones más amplias que las personales). Los "modelos experienciales" en la formación de los padres y madres posibilitan dichos espacios y encuentros de intercambio entre grupos de padres y madres ya que, entre todos, cuentan con un potencial de recursos y destrezas que resultan de gran utilidad (Maíquez, Rodrigo, Capote \& Vermaes, 2000; Maíquez, Blanco-Villaseñor, Rodrigo \& Vermaes, 2000; Maganto \& Bartau, 2004; Martínez, 2009; Orte, Ballester \& Mach, 2013; Rodrigo, et.al, 2000, 2006, 2008, 2010a, 2010b; Torío, Peña, Rodríguez, Fernández, Molina, Hernández \& Inda, 2013). Frente a un modelo académico (adquisición de conceptos sobre el desarrollo y la educación en un escenario de aprendizaje formal -padres como alumnos-) o un modelo técnico (adquisición de técnicas y procedimientos basados en la modificación de conducta, en el aprendizaje social, etc. en un escenario de aprendizaje experto -padres como expertos-), los padres y las madres se 


\section{Torío et al - Parentalidad Positiva y Formación Experiencial}

sienten activos y protagonistas en su tarea educativa y la intervención debe enfocarse hacia el desarrollo de sentimientos de confianza en su capacidad para educar a sus hijos e hijas. Este proceso no se consigue a través de charlas, sino mediante procedimientos que generen reflexión y análisis personal. El verdadero sentido de lo experiencial implica seguir un proceso de reflexión y análisis de las prácticas educativas, de las consecuencias de éstas en la vida familiar o en el desarrollo de los hijos e hijas para finalmente la verbalización de los objetivos personales de cambio.

Desde esta perspectiva, presentamos el programa "Construir lo cotidiano" (Torío, et.al., 2013a), programa de educación grupal y de modalidad experiencial, dirigido a fortalecer una parentalidad positiva. Dicho programa se centra en abordar la corresponsabilidad familiar o la maternidad y paternidad compartida con el objetivo de proporcionar la oportunidad de unas relaciones más igualitarias y nuevos modelos de socialización en la crianza y educación de los hijos e hijas, es decir, alude al hecho de que las actividades domésticas y el cuidado de las personas con quien se convive sean compartidas por personas de ambos sexos. Se busca promover un modelo educativo que ayude a los menores a crecer en la comprensión y fomentar valores de igualdad y democracia. Es necesario destacar que una condición indispensable para la participación en el programa, es la asistencia y colaboración de ambos miembros de la pareja, salvo en el caso de hogares monoparentales. El fin último, habilitar a las familias hacia el desarrollo de una parentalidad positiva en el cumplimiento de los cinco principios básicos mencionados.

El presente artículo pretende ofrecer una contribución a la tarea de la evaluación. El procedimiento de evaluación de resultados se concreta en tres momentos de un proceso planificado de evaluación del programa: un pre-test inicial, un informe valorativo final y un pos-test transcurridos, al menos, seis meses de la aplicación del mismo. Centramos la atención, en estas páginas, en la evaluación final, con objeto de presentar algunos de los resultados más destacados del programa tomando como referencia algunos de los instrumentos elaborados para tal fin: el cuestionario final del programa para madres y padres (sesión 10) y una dinámica que se realiza en dicha sesión, a través de un documento de trabajo, donde se obtienen interesantes valoraciones de las familias sobre los efectos y logros alcanzados con el programa y los cambios a corto plazo en su comportamiento en las áreas trabajadas. 


\section{Método}

\section{Muestra}

La muestra la forman 48 padres y madres con una edad promedio 41,58 (D.T.=4,98). En la Tabla 1 se puede ver la distribución de las familias en cada uno de los seis centros en los que se desarrolló el programa. El número de familias monoparentales fue 4 de un total de las 26 familias participantes. No existen diferencias en la edad según el género de los progenitores, la edad media de las madres fue de 41,12 (D.T.=4,80) y los padres 42,14 (D.T.=5,24). El nivel medio estudios es "universitarios medios". En esta variable, se hallaron diferencias estadísticamente significativas por género $(\mathrm{t}$ Student $=-2,37, \mathrm{p} \leq 0,05)$. En las madres, la distribución en esta variable era: $4 \%$ estudios primarios, $4 \%$ educación secundaria, $15 \%$ formación profesional, 35\% diplomatura universitaria, 38\% universitarios superiores y 4\% doctorado. En los padres: $13 \%$ estudios primarios, $14 \%$ educación secundaria, $27 \%$ formación profesional, $23 \%$ diplomatura universitaria y $23 \%$ universitarios superiores.

La media de hijos o hijas en los seis centros es entre 1 y 2 hijos. Respecto al sexo del primer descendiente, en tres de los centros el valor más encontrado ha sido el de los varones, y en uno, las niñas. En relación con el segundo hijo, se repite lo mismo, en tres centros el segundo descendiente con mayor frecuencia es un varón. Por lo que respecta a la edad del primer hijo y en la edad del segundo descendiente existen diferencias estadísticamente significativas como lo muestra la prueba de Kruskal Wallis $\left(\chi 2=12,18 ; \mathrm{p}<0,05\right.$ y $\chi^{2}=9,22 ; \mathrm{p}<0,05$, respectivamente $)$. 
Tabla 1.

Características de las familias evaluadas

\begin{tabular}{lccccccc}
\hline Centro & $\begin{array}{c}\text { Edad } \\
\text { media de } \\
\text { los padres } \\
\text { cuando } \\
\text { nació el } \\
\text { primer } \\
\text { hijo }\end{array}$ & $\begin{array}{c}\text { Prome } \\
\text { dio de } \\
\text { hijos/a } \\
\text { SII }\end{array}$ & $\begin{array}{c}\text { Edad } \\
\text { media } \\
\text { del } \\
\text { primer } \\
\text { hijo/hija } \\
\text { II }\end{array}$ & $\begin{array}{c}\text { Edad } \\
\text { media del } \\
\text { segundo } \\
\text { hijo/hija }\end{array}$ & $\begin{array}{c}\text { Género } \\
\text { del } \\
\text { primer } \\
\text { hijo/hija } \\
\text { II }\end{array}$ & $\begin{array}{c}\text { Género } \\
\text { del } \\
\text { Segundo } \\
\text { hijo/hija } \\
\text { II }\end{array}$ & $\begin{array}{c}\text { Género } \\
\text { del } \\
\text { tercer } \\
\text { hijo/hija } \\
\text { II }\end{array}$ \\
\hline Centro 1 & 37,50 & 1 & 6 & ------------ & Niño & ------- & ------- \\
\hline $\begin{array}{l}\text { Centro 2 } \\
\text { III }\end{array}$ & 31 & 1 & 6,40 & 6 & Niño & Niño & Niña \\
\hline Centro 3 & 38 & 2 & 6,25 & 4 & Niña & Niño & -------- \\
\hline Centro 4 & 32,60 & 1 & 9,50 & 8 & Niño & Niña & -------- \\
\hline Centro 5 & 29,20 & 2 & 4,50 & 0,60 & Niño & Niña & -------- \\
\hline Centro 6 & 33,89 & 2 & 8,44 & 3,48 & Niño & Niño & Niño \\
\hline III
\end{tabular}

Nota: I. La prueba de Kruskal-Wallis muestra diferencias estadísticamente significativas por centro educativo, en estas variables: edad comienzo paternidad $(\chi 2=20,01 ; p \leq 0,001)$, edad primer hijo $(\chi 2=12,18 ; p \leq 0,05)$, edad segundo hijo $(\chi 2=9,22 ; p \leq 0,05)$, II. Se muestra el valor de la Moda en esta variable. III. Había una familia en cada centro con tres hijos.

\section{Instrumento}

Para el desarrollo de estas páginas, se utilizarán los datos obtenidos de dos instrumentos de evaluación diseñados específicamente para el programa y aplicados durante la realización de la sesión 10 -sesión de cierre-:

1. "Cuestionario de evaluación final para padres y madres participantes en el programa" (Torío et al., 2013). Este instrumento ha sido diseñado por los miembros del equipo con el objetivo de tener un indicador más para la evaluación sumativa del programa y más concretamente, de la satisfacción de las familias participantes. Está formado por 19 preguntas, de las cuales 8 son cerradas -cinco con respuestas de no (1) y sí (2) y tres con cuatro alternativas de respuesta: nunca (1), a veces (2), casi siempre (3) y siempre (4)- y las otras 11 son abiertas en las que se requiere justificar y ampliar la información de las respuestas cerradas. El interés de dicho instrumento es conocer, por un lado, si los participantes consideran que se han producido cambios en la imagen de ser padres y 
madres, en los modos de educar, si ha mejorado el reparto de las tareas domésticas y la participación de los hijos en las tareas familiares, resolución de conflictos y en el establecimiento de una buena comunicación. Asimismo, por otro lado, valoran la metodología y recursos del programa: si las sesiones han sido comprensibles, si consideran interesantes las actividades trabajadas en la sesiones presenciales o solicitadas para realizar en casa, la validez que otorgan a las "guías de padres y madres", las actividades que más y menos le han gustado. En este instrumento también parecía interesante que las familias realizaran una estimación de la perdurabilidad de los cambios logrados e, igualmente, que emitieran una valoración crítica global sobre los aspectos que, a su juicio, deberían ser cambiados en su desarrollo. Finalmente, se solicita a los padres y madres que indiquen si recomendarían este programa a otras familias.

2. Documento de trabajo para la dinámica de la sesión 10, una actividad que pretende ayudar a reconocer los obstáculos que encuentran las familias en los procesos de cambio y ayudar a formular sus propuestas de mejora. Las preguntas señaladas en el documento son las siguientes. “QQué ha cambiado en las relaciones familiares? ¿Qué obstáculos nos hemos encontrado al iniciar los procesos de cambio? ¿Tenéis previsto alguna acción de mejora? ¿Cuál?”. Se trata de ayudar a verbalizar las dudas y dificultades que han encontrado y concretar en qué aspectos quieren mejorar.

\section{Procedimiento}

La aplicación y evaluación del programa se puso en marcha durante el curso académico 2010/2011 en diferentes centros educativos en los niveles de Educación Infantil y Educación Primaria del Principado de Asturias y sigue vigente en la actualidad (un total de 7 centros educativos han participado de esta experiencia). Para el inicio de la implementación del programa, el equipo de investigación se puso en contacto con cada uno de los centros escolares, vía correo postal. En el documento enviado se ofrecía la posibilidad de trabajar el programa de manera gratuita y ajustándose a las preferencias horarias de las familias participantes. El contacto inicial en la mayoría de los casos ha sido realizado a través de las Asociaciones de 
Madres y Padres de las respectivas instituciones. En aquellos centros en los que se obtuvo una respuesta positiva, se realizó una jornada de presentación a todas las familias interesadas. En dicha sesión, se repartieron trípticos informativos y se explicó con un mayor grado de detalle el funcionamiento del programa, sus contenidos, la dinámica de trabajo así como los requisitos para la participación. Las condiciones metodológicas propuestas -grupos de no más de 9/11 personas- supone cierto grado de dificultad para aumentar el tamaño de la muestra con cierta rapidez.

Una vez implementado en los centros, cuando el programa se encuentra en el desarrollo de la décima sesión y, por tanto, a punto de finalizar, se procede a la evaluación sumativa y final del mismo a través de la dinámica de la sesión y, al finalizar la misma, la aplicación del "Cuestionario de evaluación final para padres y madres participantes en el programa".

En lo que atañe al análisis de los datos, se realiza en base tanto a un análisis cuantitativo como cualitativo.

\section{Resultados}

\section{Resultados del Cuestionario de Evaluación Final para Padres y Madres Participantes}

Para poder analizar la relación entre las variables que evalúa el instrumento de medida se consideró emplear un modelo log-lineal jerárquico. Este método estadístico permite analizar la relación entre más de dos variables categóricas considerando en un primer momento todas las relaciones posibles entre las variables y, a continuación, va eliminando en fases sucesivas, aquellos efectos cuya supresión no disminuye significativamente el ajuste del modelo.

El primer modelo que se consideró ha sido analizar la relación entre: "secuencia de las sesiones comprensibles y coherentes" * "contenidos en cada sesión son provechosos para la vida familiar"* "dinámicas o actividades de las sesiones son provechosas para mi vida familiar" * "tengo expectativas que los cambios se prolonguen en el tiempo". El modelo que ha resultado significativo es el de orden $2\left(\mathrm{~K}=2, \mathrm{X}^{2}=89.09\right.$, $\left.\mathrm{p} \leq .001\right)$ (ver Tabla 2). 
REMIE -Multidisciplinary Journal of Educational Research, 5(3)305

Tabla 2.

Modelos resultantes de orden 2

\begin{tabular}{clccc}
\hline Efectos significativos & $\mathrm{X}^{2}$ & $\mathrm{~g} .1$. & $\mathrm{p}$ \\
\hline$\bullet$ & $\begin{array}{l}\text { contenidos en cada sesión son provechosos para la vida } \\
\text { familiar * dinámicas o actividades de las sesiones son } \\
\text { provechosas para mi vida familiar" }\end{array}$ & 31,42 & 16 & 0,01 \\
$\begin{array}{l}\text { secuencia de las sesiones comprensibles y coherentes * } \\
\text { dinámicas o actividades de las sesiones son provechosas } \\
\text { para mi vida familiar } \\
\text { dinámicas o actividades de las sesiones son provechosas } \\
\text { para mi vida familiar"* tengo expectativas que los cambios } \\
\text { se prolonguen en el tiempo }\end{array}$ & 24,78 & 4 & 0,04 \\
\end{tabular}

Nota $=$ La probabilidad de ajuste al modelo es $\mathrm{X}^{2}=17,98, \mathrm{p}>0,05$.

A continuación se analiza en detalle los efectos de segundo orden. La interacción entre los contenidos de las sesiones y las dinámicas con las que se trabajan es un efecto a tener en cuenta en la puesta en marcha del programa, el Coeficiente de contingencia igual a $0,79(\mathrm{p} \leq 0,001)$. La relación entre la secuenciación de las sesiones y la percepción de los participantes sobre su influencia en la vida familiar son factores que han funcionado positivamente en el programa (Coeficiente de contingencia $=0,82, p \leq 0,001$ ). La estructura en cómo se han trabajado las sesiones tiene relación significativa con las expectativas de los y las participantes respecto a la prolongación en el tiempo de los cambios conseguidos con el programa (Coeficiente de contingencia $=0,72, \mathrm{p} \leq 0,001$ )

Se consideró analizar si existían diferencias según el género de los padres, $X^{2}=37,95, p>0,05$. En el caso de los padres se obtuvo el siguiente efecto significativo: "secuencia de las sesiones comprensibles y coherentes" y "tengo expectativas que los cambios se prolonguen en el tiempo" $\left(\mathrm{X}^{2}=13,14, \mathrm{p} \leq .01\right)$. (Coeficiente de contingencia $\left.=0,71 ; \mathrm{p} \leq 0,001\right)$.

En las madres, probabilidad de ajuste al modelo $\mathrm{X}^{2}=27,91, \mathrm{p}>0,05$, se encuentran dos efectos significativos: hay asociación significativa entre "secuencia de las sesiones comprensibles y coherentes" y "tengo expectativas que los cambios se prolonguen en el tiempo" $\left(\mathrm{X}^{2}=14,09\right.$, $\mathrm{p} \leq .01$ ), (Coeficiente de contingencia $=0,71 \mathrm{p} \leq 0,001)$ y "dinámicas $\mathrm{o}$ actividades de las sesiones son provechosas para mi vida familiar"* tengo 
expectativas que los cambios se prolonguen en el tiempo $\left(X^{2}=49,04\right.$, $\mathrm{p} \leq .001)$, (Coeficiente de contingencia $=0,72 \mathrm{p} \leq 0,001)$.

El segundo modelo considerado para analizar es la relación entre "cambios en las imágenes de maternidad y paternidad" * "modos de educar"* "reparto de tareas domésticas" *"participación de los hijos e hijas en las tareas familiares" * "resolución de conflictos" * "establecimiento de una buena comunicación". El modelo que ha resultado significativo es de orden $1\left(\mathrm{~K}=1, \mathrm{X}^{2}=57.15, \mathrm{p} \leq .001\right)$.

Los cambios que mayormente han indicado los y las participantes en el programa, en la última sesión del mismo, ha tenido que ver con "el establecimiento de una buena comunicación", las madres muestran un $77 \%$ de la participantes, en los padres sube a un $82 \%$ los que indican que han mejorado. En la dimensión "resolución de conflictos" las madres dicen haber mejorado en un 36\%. En los padres desciende este porcentaje, $23 \%$ de mejoría. En "la participación de los hijos e hijas en las tareas familiares", $20 \%$ de las madres indican que sí han observado cambios y en los padres el $23 \%$. "Reparto de tareas domésticas", las madres indican un 23\% de mejoría y los padres el 18\%. En los "modos de educar", las madres indican en un $31 \%$ que el programa ha contribuido a establecer modelos democráticos $\mathrm{y}$ los padres en un $18 \%$. La imagen del ser padre y madre es la dimensión donde los y las participantes afirman, en la última sesión, donde los porcentajes son más bajos, en el caso de las madres en un $8 \%$ y en los padres en un $9 \%$.

\section{Documento de trabajo para la dinámica de la sesión de cierre}

Los cambios van orientados a modificar aspectos de su mentalidad y creencia que permitan dar un nuevo sentido a sus experiencias familiares. En el intento de señalar al menos dos cambios, se indica que los principales logros han sido en aspectos relativos, en primer lugar, a la comunicación. Así, manifiestan, por ejemplo, "Me fijo más en la manera en que me comunico con mi hijo", "Estamos más dispuestos a comunicarnos tranquilamente. Intentamos guardar más la calma", "Usamos más los mensajes que expresan nuestros sentimientos ante algo que nos moleste", "Soy más consciente de la necesidad de escuchar", "Más diálogo, menos desprecios", etc. También se indican logros en aspectos relativos a la 
resolución de los conflictos y su negociación: "No reñir tanto y más negociar", "Resolver los conflictos. Tener más paciencia con los hijos", "Enfocar los conflictos de forma diferente". De igual modo, el establecimiento de las normas es un tema de gran de gran interés y atención que ha provocado cambios en la vida cotidiana: "Entiendo la importancia de establecer normas claras", "Mi firmeza en el comunicación de la norma", "Establecemos normas con mayor coherencia". Finalmente, se constatan cambios en el reparto del trabajo doméstico: "Mejora en el trabajo doméstico", "Participación de los hijos en las tareas domésticas", "Más colaboración", "Mejor comprensión del reparto de tareas domésticas", "Hemos hecho más hincapié en las tareas de nuestros hijos en la casa". Es valorada como muy interesante la presencia de ambos miembros de la pareja, condición a la que no hemos renunciado en la captación de familias, ya que es fundamental para provocar cambios en la dinámica familiar y más, si cabe, en la temática que nos ocupa.

Respecto a los obstáculos que encuentran a la hora de iniciar procesos de cambio, se destacan "la paciencia", "el estado de ánimo (a veces no hay tiempo, prisa, decides esperar o iniciar otro día...)", "el autocontrol", "los hábitos (nos cuesta romper con lo que venimos haciendo siempre, formas de ser, carácter)", "cosmovisiones diferentes: yo lo quiero cambiar y él no", ponerse en el lugar del otro". La familia es una comunidad solidaria en la que sus miembros deben estar dispuestos a ceder y recibir.

Sus respuestas sobre las posibles acciones de mejora y compromisos futuros, sus opiniones nos transmiten el interés de seguir transfiriendo los conocimientos aprendidos a la vida cotidiana y la posibilidad de recurrir a las Guías de padres y madres facilitadas al finalizar cada una de las sesiones. Hemos de destacar respecto a la percepción sobre la perdurabilidad de dichos cambios, en general, la mayoría de las familias tienen expectativas positivas a largo plazo sobre la estabilidad en los cambios conseguidos con el programa.

\section{Discusión}

La experiencia de diseño, implementación y evaluación del programa "Construir lo cotidiano" ha sido, sin duda, exitosa. Los padres y madres del programa han iniciado un proceso de cambio en su dinámica familiar así 
como han reafirmado aspectos de su tarea educativa (Torío, Peña, Rodríguez, Fernández \& Molina, 2010; Torío, Peña \& Hernández, 2012; Torío, Peña, Fernández, Inda \& Rodríguez, 2013; Torío, Peña, Inda, Fernández \& Rodríguez, 2015; Torío, Inda \& Fernández, 2015). Se puede afirmar que las relaciones y la dinámica de grupo entre padres y madres y profesionales es positiva tanto por la asistencia como por la valoración explícita que se hace de la posibilidad de participar y debatir en relación a temas educativos. Consideramos que la participación e implicación de las familias participantes en el mismo, facilita el principio de aprendizaje experiencial: "llevar a cabo una reconstrucción del conocimiento episódico cotidiano en un escenario sociocultural. (...) se tienen en cuenta las creencias o las ideas previas de los padres y las madres, ya que a partir de éstas y de las situaciones cotidianas, y mediante un proceso inductivo de construcción del conocimiento cotidiano-experiencial, van a ir construyendo su conocimiento" (Martín-Quintana, Byme, Máiquez, Rodríguez Ruiz, Rodrigo \& Rodríguez Suárez, 2009, p. 127). Se sienten escuchados y comprendidos, crean lazos de amistad entre las personas participantes, y se disminuye el sentimiento de culpa que tienen muchos padres y madres al constatar que otros tienen las mismas inquietudes e, incluso, problemáticas. Algunos rasgos de la personalidad de los padres y madres asociados a la buena parentalidad positiva, y reforzados en el programa, son los siguientes (Loizaga Latorre, 2011): flujo o sensación vital de fluidez, creatividad con dosis de incertidumbre, inteligencia emocional para poder entender a los otros y a uno mismo y la resiliencia o capacidad de sobreponerse a los acontecimientos adversos y traumáticos que seguro vamos a vivir. En síntesis, se ha cubierto satisfactoriamente uno de los objetivos centrales del programa: poner en marcha procesos de socialización para que en el seno de los grupos de trabajo se produzcan cambios en los esquemas mentales estereotipados de género en materia de corresponsabilidad.

El diseño de la estructura del programa es una de las variables que se ha tenido en cuenta puesto que permite percibir efectos positivos o no del mismo. Yendo por partes, en primer lugar, los contenidos del programa y el modo de implementarlo con sus dinámicas y las actividades, son valorados positivamente por los participantes, siendo las madres las que más hincapié realizan en esta relación. Por otro lado, los factores del programa que tienen que ver con la estructura y la secuenciación de las sesiones es valorada igualmente pues permite analizar los cambios en los distintos participantes y 
fortalecer los mismos. Estos datos nos permiten captar que no solo cumplen un fin formativo, sino que se alcanza el nivel educativo para el que fueron diseñadas. Recordemos que el diseño del programa se efectuó pensando en una intervención durante la infancia ya que las interacciones sostenidas por los padres y madres y sus hijos e hijas alrededor de las tareas de socialización pueden servir para construir un estilo interactivo con cada una de las partes (madre/hijo o hija y padre/hijo o hija) para asentar un patrón relacional antes de llegar a la adolescencia.

Estos pequeños cambios de proceso lento tienen que ver mucho, siendo realistas, con la tipología de las familias que han participado en el programa. En los programas dirigidos a familias con situaciones multiproblemáticas (Carcelén, Senabre, Morales \& Romero, 2010; De Paúl \& Arrabarrena, 2003; Goméz, Cifuentes \& Ortún, 2012; Gómez, Muñoz \& Haz, 2007; Martínez, 2009; Orte, Touza, Ballester \& March, 2008; Orte, Ballester \& March, 2013; Rodrigo et al, 2008, 2010) puede parecer que presentan resultados exitosos. Dichas familias presentan múltiples focos estresores que colapsan el equilibrio del sistema familiar y, en gran medida, la experiencia consigue disminuir los niveles de riesgo familiar para el desarrollo infantil desde un nivel peligroso a un nivel ubicado por debajo de los umbrales considerados de riesgo (Gómez, Cifuentes \& Ortún, 2012). Cuatro son los ejes que se manifiestan entrelazados en las problemáticas familiares (Gómez, Muñoz \& Haz, 2007): polisintomatología (cadena de problemas y factores de estrés); desorganización (estructura caótica y comunicación disfuncional); abandono de las funciones parentales (incompetencia y negligencia) y aislamiento (exclusión).

Por otro lado, los programas para familias en situación de riesgo y multiproblemáticas (Barth, 2009; Gómez, Cifuentes \& Ortún, 2012) destacan el incremento significativo de los resultados cuando se tratan de modelos mixtos centro/hogar (se incluye visita domiciliaria) y se ofrecen atenciones individuales y grupales combinadas y espacios para practicar "en vivo", "in situ" nuevas habilidades con los hijos e hijas, focalizándose en la interacción padres/hijos, la respuesta parental y la comunicación emocional en la familia. El Programa de Parentalidad Positiva "Triple P" (Sanders, Markie-Dadds \& Turner, 2003) diseñado en la Universidad de Queesland, Australia e implementado en más de 20 países en el mundo, mostró que genera cambios positivos en habilidades parentales, problemas conductuales y bienestar parental, variando los resultados en función de la intensidad de 


\section{Torío et al - Parentalidad Positiva y Formación Experiencial}

su intervención (nivel 1 al 5), con mayor tamaño de efecto a mayor intensidad y según la modalidad de entrega (individual, grupal y autoadministrada) con mejores resultados en intervenciones personalizadas (Gómez, Cifuentes \& Ortún, 2012; Nowak \& Heinrichs, 2008). Podemos incluso ir más allá, los cambios positivos y la minimización de los factores de riesgo tienen mayor recorrido e impacto si abarcamos el conjunto de la familia, programas como el "Programa de Competencia Familiar" (PCF, adaptación española del Strengthening Families Program de Kumpfer, de Orte et. al, 2008, 2013) que complementa la formación de los padres con la formación de los hijos, atendiendo a aspectos que influyen directamente sobre la educación integral de todos los miembros de la misma.

En nuestro caso, los cambios son más lentos, modestos y restringidos pero no menos importantes. Son familias que atienden de forma adecuada a las necesidades de crianza de sus hijos en la que en mayor o menor medida ambos miembros de la pareja están implicados en ese proceso y comparten actitudes, valores e, incluso, normas. Sin embargo, perciben que hay un campo amplio de mejora que no solo va a favorecer los procesos de crianza sino la dinámica familiar cotidiana y la comunicación entre sus miembros, especialmente, en los aspectos educativos. Descubren que, profundizando en una estructura familiar menos estereotipada por género y con roles menos diferenciados, las cosas pueden funcionar mejor y ser más gratificante para ellos mismos.

Algunas de las familias que han participado en el programa "Construir lo cotidiano" han reclamado atenciones individualizadas relativas a problemáticos específicas o para practicar habilidades con los hijos e hijas (es decir, la combinación de un trabajo grupal y la atención a la pareja). No se ha podido atender este reclamo por varias razones: la primera y quizá más relevante es que el programa no se ha construido con este tipo de objetivos. La idea central del programa es facilitar un funcionamiento autónomo y responsable en el afrontamiento de la vida familiar, partiendo de lo que hacen diariamente (no de lo que debería ser). Así mismo, posibilita ensayar el cambio en la vida real y enmarcar éste dentro de un proyecto vital significativo, incrementando los sentimientos de control de su vida y optimismo vital, todos ellos, componentes básicos del aprendizaje experiencial (Rodrigo, Máiquez \& Martín, 2010b).

A parte de la razón señalada, también, se optó desde un principio por no abordar cuestiones de carácter terapéutico familiar puesto que exigiría 
intervenciones individualizadas con una dinámica y un proceso que rompe la estructura del programa diseñado. No descartamos, en un futuro, complementar las dinámicas grupales de este trabajo educativo familiar con labores complementarias de diagnóstico e intervención individualizada.

En síntesis, en estos procesos de cambio, se ofrecen a las familias oportunidades de aprendizaje que terminan posibilitando la reconstrucción/optimización del ejercicio de la parentalidad.

\section{Referencias}

Barth, R. (2009). Preventing Child Abuse and Neglect with Parent Training:

Evidence and Oppotunities. The Future of Children, 19(2), 95-118. doi:10.1353/foc.0.0031

Carcelén, R., Senabre, I., Morales, J.L., \& Romero, F.J. (2010). ¿Cómo puedo proteger a mis hijos de las drogas?: Una experiencia de prevención de drogas en familia. Revista Española de

Drogodependencias, 35(1), 92-97. Disponible en http://mobiroderic.uv.es/bitstream/handle/10550/22439/v35n1_7.pdf?se quence $=1 \&$ is Allowed $=y$

Center for Parenting and Research. (2006). Effective parenting capacity assessment: key issues. Disponible en http://www.community.nsw.gov.au/docswr/_assets/main/documents/res earchnotes_parenting_keyissues.pdf

Consejo de Europa. (2006). Recomendación REC (2006)19 del Comité de Ministros sobre políticas de apoyo a la parentalidad positiva.

Estrasburgo: Consejo de Europa.

Consejo de Europa. (2007). Parenting in contemporany Europe: a possitive approach. Estrasburgo: Consejo de Europa.

De Paúl, J., \& Arruabarrena, I. (2003). Evaluation of a treatment program for abusive and high-risk families in Spain. Child Welfare League of America, 82, 413-442.

Gómez, E., Muñoz, M., \& Haz, A.M. (2007). Familias multiproblematicas y en riesgo social: características e intervención. Psyke, 16(2)43-54. doi:10.4067/S0718-22282007000200004

Gómez, E., Cifuentes, B., \& Ortún, C. (2012). Padres competentes, Hijos protegidos: Evaluación de resultados del Programa "Viviendo en 
Familia”. Psychosocial Intervention, 21(3), 259-271. doi:10.5093/in2012a23

González Sánchez, R. (Coord). (2013). Queriendo se entiende la familia. Guía de intervención sobre parentalidad positiva para profesionales. Madrid: Save the Children.

Loizaga Latorre, F. (2011). Parentalidad positiva. Las bases de la construcción de la persona. Educación social, 49, 70-88.

Maganto, J.M., \& Bartau, I. (2004). Corresponsabilidad familiar

(COFAMI): Fomentar la cooperación responsabilidad de los hijos.

Madrid: Pirámide.

Maíquez, Ma L, Rodrigo, Ma J., Capote, C., \& Vermaes, I. (2000). Aprender en la vida cotidiana. Un programa experiencial para padres. Madrid:

Visor.

Maíquez Chaves, Mª L., Blanco-Villaseñor, A., Rodrigo López, Mª J., \& Vermaes, P.R. (2000). La evaluación de la eficiencia en la intervención familiar: generalizabilidad y optimización del Programa experiencial para padres. Psicothema, 12(4), 533-542. Disponible en http://www.psicothema.com/pdf/368.pdf

March Cerdá, M.X., \& Orte Socias, C. (Coords). (2014). La pedagogía social y la escuela. Los retos socieducativos de la institución escolar en el siglo XXI. Barcelona: Octaedro.

Martín-Quintana, J.C., Byme, S., Máiquez, Ma L., Rodríguez, B., Rodrigo, $\mathrm{M}^{\mathrm{a}}$ J., \& Rodríguez, G. (2009). Programas de educación parental.

Intervención Psicosocial, 18(2), 121-133. Disponible en

http://scielo.isciii.es/scielo.php?pid=S1132-

05592009000200004\&script=sci_arttext

Martínez González, R.A. (2009). Programa-Guía para el desarrollo de competencias emocionales, educativas y parentales. Madrid: Ministerio de Sanidad, Política Social.

Moreno, E. (2010). Políticas de infancia y parentalidad positiva en el marco europeo. Papeles Salmantinos de Educación, 14, 17-28. Disponible en http://summa.upsa.es/pdf.raw?query=id:0000030627\&page=1\&lang=es

Nowak, Ch., \& Heinrichs, N. (2008). A Comprehensive metaanalysis of triple P-Positive Parenting Program using hierarchical linear modeling: Effectiveness and moderating variables. Clinical Child and Family Psychological Review, 11, 114- 144. doi:10.1007/s10567-008-0033-0 
Orte, C., Touza, C., Ballester, Ll., \& March, M.X. (2008). Children of drugdependent parents: prevention programme outcomes. Educational Research, 50(3), 249-260. doi:10.1080/00131880802309390

Orte, C., Ballester, L., \& March, M.X. (2013). El enfoque de la competencia familiar. Una experiencia de trabajo socioeducativo con familias.Pedagogía Social. Revista Interuniversitaria, 21, 13-37.

Rodrigo, Mª J., Capote, C., Máiquez, M.L., Martín, J.C., Rodríguez, G., Guimera P., \& Peña, M. (2000). Manual del Programa Apoyo Personal y Familiar. Santa Cruz de Tenerife: Fundación ECCA.

Rodrigo, M.J., Correa, A.D., Maíquez, Mª L., Martín, J.C., \& Rodríguez, G. (2006). Family preservation services in Canary Island: Predictors of the efficacy of a Parenting program for families at-risk of social exclusion. European Psychologist, 11(1), 57-70. doi:10.1027/1016-9040.11.1.57

Rodrigo, Mª J., Máiquez, M.L., Byme, S., Rodríguez, B., Martín, J.C., Rodríguez, G., \& Pérez, L. (2008). Programa Crecer Felices en familia. Programa de apoyo psicoeducativo para promover el desarrollo infantil. Valladolid: Junta de Castilla y León.

Rodrigo, Ma J., Maíquez, Mª L., \& Martín, J. C. (2010a). Parentalidad positiva y políticas locales de apoyo a las familias. Orientaciones para favorecer el ejercicio de las responsabilidades parentales desde las corporaciones locales. Madrid: Federación Española de Municipios y Provincias (FEMP).

Rodrigo, Mª J., Maíquez, Mª L., \& Martín, J. C. (2010b). La educación parental como recursos psicoeducativo para promover la parentalidad positiva. Madrid: Federación Española de Municipios y Provincias (FEMP).

Rodrigo, Ma J., Martín, J.C., Máiquez, M.L., Álvarez, M., Byme, S., González, A., Guerra, M., Montesdecoa, M.A., \& Rodríguez, B. (2010). Programa Vivir la adolescencia en familia. Programa de apoyo psicoeducativo para promover la convivencia familiar. Toledo: Junta de Comunidades de Castilla La Mancha.

Sanders, M.R. (2008). Triple P-Positive Parenting Program as a public health approach to strengthening. Journal of family psychology, 22(3), 506-517. doi:10.1037/0893-3200.22.3.506

Sanders, M. R., Markie-Dadds, C., \& Turner, M.T. (2003). Theoretical, scientific and Clinical Foundations of the Triple P-Positive Parenting Program: A population approach to the promotion of parenting 
competence. Parenting Research and Practice Monograph, 1. University of Queensland. Australia: Parenting and Family Support Centre.

Torío, S., Peña, J.V., Rodríguez, M.C., Fernández, C.M., \& Molina, S. (2010). Hacia la corresponsabilidad familiar: "Construir lo cotidiano". Un programa de educación parental. Educatio Siglo XXI, 28(1), 85-108. Disponible en http://revistas.um.es/educatio/article/view/109741

Torío, S., Peña, J.V., \& Hernández, J. (2012). Primeros resultados de la aplicación y evaluación de un programa de educación parental: "Construir lo cotidiano". Teoría de la Educación. Educación y Cultura en la sociedad de la información, 13(2), 343-368. Disponible en

http://campus.usal.es/ revistas_trabajo/index.php/revistatesi/article/vi ew/9012/9256

Torío, S., Peña, J.V., Rodríguez, C., Fernández, C.M., Molina, S., Hernández, J., \& Inda, M. (2013). Construir lo cotidiano: un programa de educación parental. Barcelona, Octaedro.

Torío, S., Peña, J.V., Fernández, C.M., Inda, Mª M., \& Rodríguez, C.M. (2013). Pervivencias, fortalecimientos y cambios de las prácticas educativas familiares. Paper presented at the Strengthening Family Strategy Online Conference. University of Balearic Island (Spain), $7^{\text {th }}$ $20^{\text {th }}$ December 2013.

Torío, S., Peña, J.V., Inda, M M M., Fernández, C. Ma , \& Rodríguez, Mª C. (2015). Evaluation of the Building Everyday Life positive parenting programme. Journal of Children's Services, 10(2), pp. 173-184. doi:10.1108/JCS-07-2014-0035

Torío, S., Inda, M $^{\mathrm{a}}$ M., \& Fernández, C.M. (en prensa). Evaluación de un programa experiencial de educación parental: la perspectiva de los educadores como agentes de cambio. Aula Abierta.

Vargas-Rubilar, J., \& Arán-Filippetti, V. (2014). Importancia de la parentalidad para el desarrollo cognitivo infantil: una revisión teórica. Revista Latinoamericana de Ciencias Sociales, Niñez y Juventud, 12(1), 171-186. doi:10.11600/1692715x.1219110813 
Susana Torío es Profesora Titular en el Departamento de Ciencias de la Educación de la Educación de la Universidad de Oviedo.

José Vicente Peña Calvo es Catedrático en el Departamento de Ciencias de la Educación de la Universidad de Oviedo.

Omar García-Pérez es Profesor Ayudante Doctor en el Departamento de Ciencias de la Educación de la Universidad de Oviedo

Contacto: Susana Torío, Facultad de Formación del Profesorado y Educación (Despacho 307) C/ Aniceto Sela, s/n. 33005 Oviedo, España, Despacho 307. Tlfn: +34-985-102883

Email: storio@uniovi.es 